\title{
A GENERAL FORMULA FOR DISPERSIVE AND RESOLVING POWERS.
}

By L. P. Sieg.

$\mathrm{T}^{\mathrm{n}}$ HE derivation of expressions for the resolving powers of prisms and diffraction gratings, as found in most works on optics, usually follow in a general way Rayleigh's ${ }^{1}$ method, while Michelson's ${ }^{2}$ proof is usually used for the echelon grating. It was suggested to the writer some time ago by C. V. Kent, of Carleton College, that a single general formula for the resolving power of any instrument of this kind might be developed which would include each of the above three as a special case. The following treatment which leads to such a formula will possibly be of interest to teachers of optics, not only on account of its comparative simplicity, but also because it shows how closely connected these three forms of spectroscopic apparatus are. As far as the writer knows no such general formula has previously been published.

In Fig. I, let $A B C$ be the intersection with the plane of the paper, of a portion of a plane wave front. Let $B C D E$ be the base of a rectangular

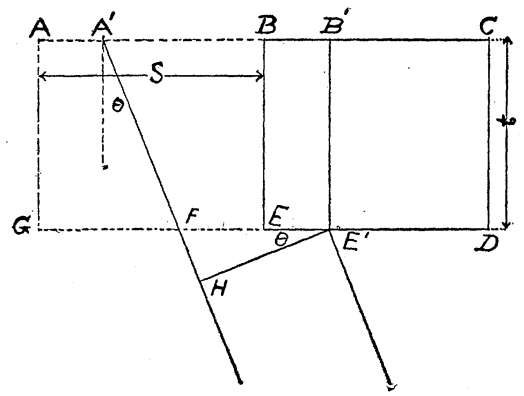

Fig. 1. prism of a transparent medium of index $\mu_{1}$. The altitude of the prism is of no concern. Let this prism be immersed in a medium of index $\mu_{2}$, of which a like prism of base $A B E G$ is considered. The intersections of the front faces of these prisms with the plane of the paper are $B C$ and $A B$, of equal length $s$. Let the depth of the prisms be $t$. Take $A^{\prime}$ and $B^{\prime}$ as corresponding

1 " Wave-Theory," Ency. Brit., Vol. XXIV. "Scientific Papers," III., 47.

2 “" The Echelon Spectroscope," Astrophys. Jour., 8, p. 36, I898. 
[Vol. XXYII.

points of the sources, i. e., $A A^{\prime}$ equals $B B^{\prime}$. Then $A^{\prime} B^{\prime}$ is equal to $s$. Consider the portion of any wave front $H E^{\prime}$ diffracted through such an angle $\theta$, that the phase difference between $H$ and $E^{\prime}$ is a multiple of $2 \pi$. In general this is not the original wave front. The geometrical path difference of the extreme portions of the wave is $\pm s \theta$, depending on the sign of $\theta$. The physical path difference is $\mu_{1} t-\mu_{2}(t \pm s \theta)$. Usually the second medium is air and taking the index for air as practically unity, and independent of the wavelength,

$$
\mu_{1} t-(t \pm s \theta)=m \lambda
$$

For the present taking only the negative value of $\theta$, we have for the dispersive power, writing $\mu$ for $\mu_{1}$,

$$
\frac{d \theta}{d \lambda}=\frac{m}{s}-\frac{t}{s} \frac{d \mu}{d \lambda}
$$

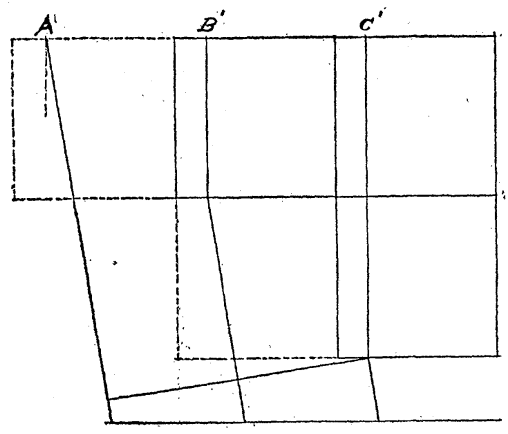

Fig. 2.

The extension of the above relation to three sources is readily seen from Fig. 2, where the dotted lines represent the necessary portions of the air medium to give the equal width of sources, and $A^{\prime}, B^{\prime}$ and $C^{\prime}$ are corresponding points. The validity of the proof is in no way affected by supposing equal and corresponding portions of the adjoining sources to be made opaque. For the resolving power, from equation (2),

$$
\frac{\lambda}{d \lambda}=\frac{\lambda}{d \theta}\left(\frac{m}{s}-\frac{t}{s} \frac{d \mu}{d \lambda}\right)
$$

In order that two images of a rectangular slit, separated by $d \theta$, may be resolved, $d \theta$ must never be less than $\lambda / e$, where $e$ is the effective breadth of the beam.

At the limit we can put $d \theta$ equal to $\lambda / e$ and obtain for the resolving. power

$$
\frac{\lambda}{d \lambda}=e\left(\frac{m}{s}-\frac{t}{s} \frac{d \mu}{d \lambda}\right)
$$


Applying equation (4) to a grating,

$$
\frac{d \mu}{d \lambda}=0 \quad \text { and } \quad \frac{\dot{\lambda}}{d \lambda}=e \cdot \frac{m}{s} .
$$

Multiplying by $n / n$, where $n$ represents the number of pairs of corresponding sources, $A B$ and $B C$,

$$
\frac{\lambda}{d \lambda}=\frac{e m}{s} \cdot \frac{n}{n}=m n
$$

since $n s$, the width of the grating, is equal to $e$. Applying (4) to the prism, $m=0$, and

$$
\frac{\lambda}{d \lambda}=-\frac{e t}{s} \frac{d \mu}{d \lambda}
$$

and as before multiplying by $n / n$,

$$
\frac{\lambda}{d \lambda}=-\frac{e t}{s} \cdot \frac{n}{n} \cdot \frac{d \mu}{d \lambda}=-T \frac{d \mu}{d \lambda}
$$

where $e=n s$, and $T=n t$, the thickness of the prism at the base. In this case we should have used the positive value of $s \theta$, Eq. (I), so that in this case

$$
\frac{\lambda}{d \lambda}=T \frac{d \mu}{d \lambda}
$$

From our knowledge of $d \mu / d \lambda$ we know that the sign of the above expression is intrinsically negative. Fig. 3 shows how a pile of infinitesimally thin plates would build up a prism. It shows at once that a prism is an echelon grating with an infinite number of steps, each of infinitesimal breadth and thickness. Of course we can here use only the spectrum of zero order, and could find the spec-

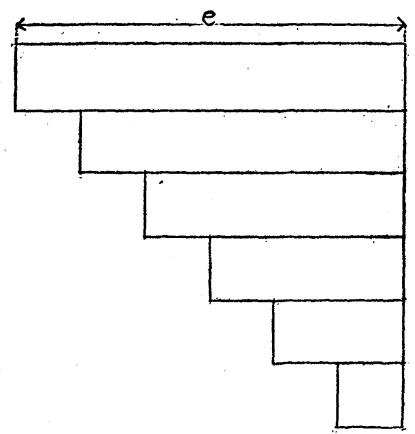

Fig. 3. trum of first order only at infinity. This is obvious since $A$ and $B$, the corresponding points of the sources, are practically coincident, so there is no possibility of obtaining a path difference of $\lambda$ in a finite field. 
Applying (4) to the echelon, we have from (I),

$$
m=\frac{(\mu-\mathrm{I}) t}{\lambda}
$$

since $s \theta$ can be neglected in comparison with $t$,

$$
\begin{aligned}
\frac{\lambda}{d \lambda} & =\frac{e t}{\lambda s}\left(\mu-\mathrm{I}-\lambda \frac{d \mu}{d \lambda}\right) \cdot \frac{n}{n} \\
& =\frac{n t}{\lambda}\left(\mu-\mathrm{I}-\lambda \frac{d \mu}{\lambda d}\right),
\end{aligned}
$$

since $n s=e$.

We may readily write the expressions for the dispersive powers, obtaining them from (2) in a manner similar to the foregoing. For the grating

$$
\frac{d \theta}{d \lambda}=\frac{m}{s} \cdot \frac{n}{n}=m n^{\prime}
$$

where $n^{\prime}=n / s$, the number of lines per unit length.

For the prism, changing the sign of the last term, as before

$$
\frac{d \theta}{d \lambda}=\frac{t}{s} \frac{d \mu}{d \lambda}\left(\frac{n}{n}\right)=\frac{T}{e} \frac{d \mu}{d \lambda},
$$

an intrinsically negative quantity. Here $n s$ equals $e$, the altitude of the base triangle of the prism.

For the echelon it is readily seen that

$$
\frac{d \theta}{d \lambda}=\frac{t}{s \lambda}\left(\mu-\mathrm{I}-\lambda \frac{d \mu}{d \lambda}\right),
$$

or with everything else constant, the dispersive power depends on the ratio of the thickness of a plate to the breadth of a step.

Physical Laboratory,

State University of Iowa, April 15, 1908. 